\title{
Appraisal of unimodal cues during agonistic interactions in Maylandia zebra
}

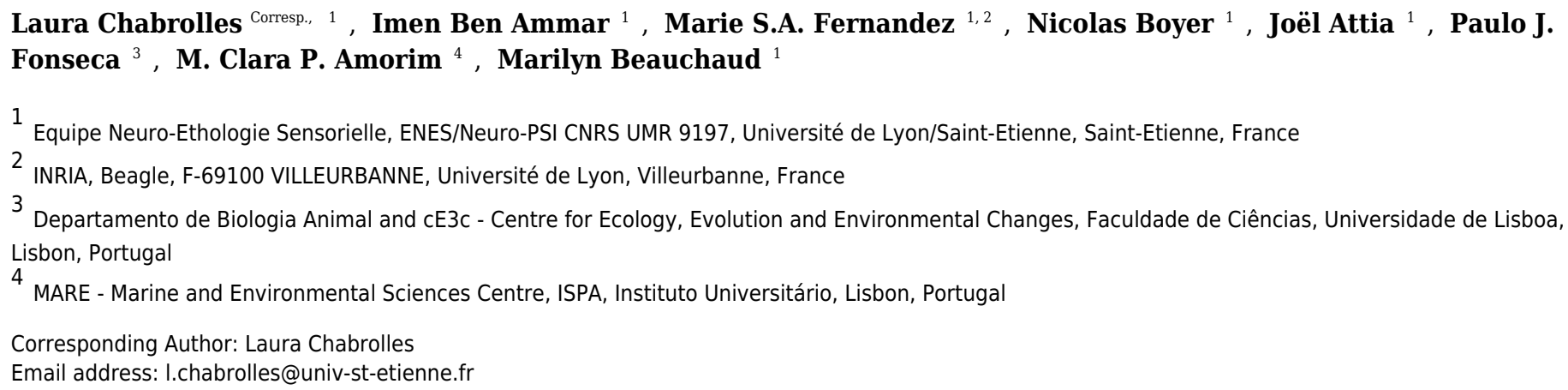

Communication is essential during social interactions including animal conflicts and it is often a complex process involving multiple sensory channels or modalities. To better understand how different modalities interact during communication, it is fundamental to study the behavioural responses to both the composite multimodal signal and each unimodal component with adequate experimental protocols. Here we test how an African cichlid, that communicates with multiple senses, responds to different sensory stimuli in a social relevant scenario. We tested Maylandia zebra males with isolated chemical (urine or holding water coming both from dominant males), visual (real opponent or video playback) and acoustic (agonistic sounds) cues during agonistic interactions. We showed that 1 ) these fish relied mostly on the visual modality, showing increased aggressiveness in response to the sight of a real contestant but no responses to urine or agonistic sounds presented separately, 2) video playback in our study did not appear appropriate to test the visual modality and needs more technical prospecting, 3) holding water provoked territorial behaviours and seems to be promising for the investigation into the role of the chemical channel in this species. Our findings suggest that unimodal signals are nonredundant but how different sensory modalities interplay during communication remains largely unknown in fish. 


\section{Appraisal of unimodal cues during agonistic interactions in Maylandia zebra}

2

3 Laura Chabrolles ${ }^{1}$, Imen Ben Ammar ${ }^{1}$, Marie S.A. Fernandez ${ }^{1,4}$, Nicolas Boyer ${ }^{1}$, Joël Attia ${ }^{1}$, 4 Paulo J. Fonseca ${ }^{2}$, M. Clara P. Amorim ${ }^{3}$ \& Marilyn Beauchaud ${ }^{1}$

5

$6{ }^{1}$ Université de Lyon/Saint-Etienne, Equipe Neuro-Ethologie Sensorielle, ENES/Neuro-PSI 7 CNRS UMR 9197, Saint-Etienne, France

8 2Departamento de Biologia Animal and cE3c - Centre for Ecology, Evolution and Environmental

9 Changes, Faculdade de Ciências, Universidade de Lisboa, Lisbon, Portugal

$10{ }^{3}$ MARE - Marine and Environmental Sciences Centre, ISPA - Instituto Universitário, Lisbon, 11 Portugal

12 4Université de Lyon, INRIA, Beagle, F-69100 VILLEURBANNE, France, Villeurbanne, France 13

14 Corresponding author:

15 Laura Chabrolles ${ }^{1}$

17 Email address: 1.chabrolles@univ-st-etienne.fr 


\section{Abstract}

20 Communication is essential during social interactions including animal conflicts and it is often a

21 complex process involving multiple sensory channels or modalities. To better understand how

22 different modalities interact during communication, it is fundamental to study the behavioural

23 responses to both the composite multimodal signal and each unimodal component with adequate

24 experimental protocols. Here we test how an African cichlid, that communicates with multiple 25 senses, responds to different sensory stimuli in a social relevant scenario. We tested Maylandia 26 zebra males with isolated chemical (urine or holding water coming both from dominant males), 27 visual (real opponent or video playback) and acoustic (agonistic sounds) cues during agonistic 28 interactions. We showed that 1) these fish relied mostly on the visual modality, showing 29 increased aggressiveness in response to the sight of a real contestant but no responses to urine or 30 agonistic sounds presented separately, 2) video playback in our study did not appear appropriate 31 to test the visual modality and needs more technical prospecting, 3) holding water provoked 32 territorial behaviours and seems to be promising for the investigation into the role of the 33 chemical channel in this species. Our findings suggest that unimodal signals are non-redundant 34 but how different sensory modalities interplay during communication remains largely unknown 35 in fish. 


\section{Introduction}

Research on animal conflict is critical to our understanding of social interactions. A common cause of conflict is the dispute for territories which can be key in acquiring food or mates (Hurd \& Enquist, 2001). Agonistic interactions during territorial contests can provoke serious injuries, but contestants can also avoid costly fights by signalling their status and performing threat displays to elicit the opponent's withdrawal (Hurd \& Enquist, 2001; van Staaden, Searcy \& Hanlon, 2011). Communication is thus fundamental in resolution of animal conflicts.

Animals communicate through multiple senses which allow them to detect and integrate information during social interactions. To better understand animal communication, we must consider all the different sensory modalities involved. It is well known that communication is a multiple sensory system (Darwin \& Prodger, 1998), but recently there is a growing interest in understanding how different sensory channels are integrated to produce adaptive behaviour (Partan \& Marler, 2005; Higham \& Hebets, 2013). A first step towards the understanding of how different modalities interact during communication, is to investigate the behavioural response in a 'cue-isolation' experiment (Partan \& Marler, 1999; Smith \& Evans, 2013). Several communication channels are known to be widely used by fish. Many authors have proposed that visual (Rosenthal \& Ryan, 2000; Dijkstra et al., 2010; Chen \& Fernald, 2011; Mellor et al., 2012), acoustic (Amorim et al., 2008; Bertucci et al., 2010; Maruska, Ung \& Fernald, 2012; Longrie et al., 2013) and chemical cues (Plenderleith et al., 2005; Martinovic-Weigelt et al., 2012; Keller-Costa, Canário \& Hubbard, 2015) are highly relevant for intraspecific communication. Additional studies revealed the importance of the lateral line system during social encounters (Butler \& Maruska, 2015). However, much remains unknown regarding the 
61 role of each sensory modality in eliciting behavioural decisions, particularly their relative

62 hierarchy during multi-modal sensory integration. For example, acoustic and chemical cues are

63 rarely analysed alone and typically need a visual trigger to elicit a behavioural response (Estramil

64 et al., 2014; Bayani, Taborsky \& Frommen, 2017). The aim of this work was to test with a 'cue-

65 isolation' experiment the prioritization of sensory modalities by investigating how fish respond

66 to isolated chemical, visual and acoustic signals in an agonistic context. It further aimed to

67 develop a bioassay to test the role of each sensory channel on behavioural decisions by assessing

68 the response to different stimulus types, e.g. video playback vs. live male stimulus.

69 African cichlids use multimodal signals to communicate during social interactions and

70 became important models to evaluate the relative importance of different sensory channels

71 (Escobar-Camacho \& Carleton, 2015). Maylandia zebra is a lek-breeding cichlid living in the

72 sediment-free rocky coast of Lake Malawi. Males of M. zebra defend territories to which they try

73 to attract females by means of conspicuous displays that involve multiples senses (Plenderleith et

74 al., 2005; Simões et al., 2008). These territories are not only used for mating but also for feeding,

75 and males defend them from other males for prolonged periods (Holzberg, 1978). In this study,

76 we exposed dominant males to the following stimuli: chemical (urine or holding water from

77 dominant males; teleost Ringer solution was used as a control), visual (a real opponent in a jar or

78 video playback), and acoustic (the playback of an agonistic sound). Based on previous studies

79 (Bertucci et al., 2010; Maruska \& Fernald, 2012), we predicted that vision would trigger direct

80 agonistic behaviours and acoustic or chemical stimuli alone would rather induce indirect

81 territorial behaviours such as increased activity and nest building.

82 


\section{Materials \& Methods}

85

Subjects and Housing Conditions

87

Seventy Maylandia zebra were purchased from Oxyfish (Verlinghem, France) and stored

89

90

91

92

93

94

95

96

97

98

99

100

101

102

103

104

105

106

The experimental aquarium $(60 \mathrm{~cm}$ x $30 \mathrm{~cm} \times 30 \mathrm{~cm})$ was placed on a vibration-insulated shelf (with a layer of wood-fibre) in a sound-proofed chamber (PRIMO Silence-Box, Tip Top Wood(C) to limit background noise. The back of the aquaria was covered on the inside with bubble wrap to break sound reflections on the tank's walls.

Each experimental tank had a filter containing active carbon, aeration, an internal heater, sand substrate, and a terracotta pot usable as a shelter by the fish, all located on the right side of the tank. Because the tested fish stayed in the experimental set up for five days, each aquarium was accompanied by a social group placed in an adjacent tank, to avoid the sense of isolation. 
107 These social groups were composed of one male and one female physically separated to avoid 108 any injuries. An underwater loudspeaker developed by Fonseca \& Alves (2012) was placed in 109 the aquarium of the tested fish and used for playing back sound stimuli. A hydrophone (Aquarian 110 Audio Products H2a-XLR, AFAB Enterprises, Anacortes, WA, USA, sensitivity: -180 dB re 1 V $111 \mu \mathrm{Pa}-1$, flat frequency response $\pm 4 \mathrm{~dB} 20 \mathrm{~Hz}-4.5 \mathrm{kHz}$ ) located in the middle of the tank, 112 monitored sound playback and registered possible sounds produced by the tested fish.

113 The hydrophone was connected to a preamplifier (Yamaha MLA8, Yamaha Music

114 France, Marne-la-Vallée, France) linked to the video capture card (Osprey-450e) of a PC that 115 synchronized audio and video signals. Behaviour was recorded using a camera (BUL520, brand, 116 Active Media Concept, Vallauris, France) positioned in front of the set-up.

117 To avoid any effect of novelty, a hermetic jar $(20 \mathrm{~cm} \times 11.7 \mathrm{~cm} \times 25 \mathrm{~cm})$ that hosted the 118 intruder fish when the visual live stimulus was presented (see below) was left in the experimental 119 tank during the five days of testing. A plastic tube (TYGON ${ }^{\circledR}$ R-3603, ID $=2.00 \mathrm{~mm}$, Saint120 Gobain Performance Plastics Verneret, La Mothe-Aux-Aulnaies, France) hooked up to the 121 hydrophone was used during chemical presentation. When no chemical stimulus was sent, a 122 piece of the same plastic tube was left during the week of experimentation. The presentation of 123 chemical stimuli was either controlled through a peristaltic pump (IPC high precision tubing 124 pump with planetary drive, ISMATEC, Switzerland) or an aquarium pump (Tecatlantis 125 EasyFlux300 and EasyFlux600, Aquatlantis, Guimarães, Portugal) adjusted with a flow rate of 126 about $1 \mathrm{~L} / 10 \mathrm{~min}$ (Kobayashi, 2002). Both pumps were located outside the sound-proofed 127 chamber (see Fig. 1 for a complete view of the set-up). 
Only males with a dominance index above 0.7 (range from 0 - subordinates, to 1 -

132 dominants) were selected for experiments or chemical sampling. To establish social hierarchies

133 between males in stock tanks, we observed the group during ten consecutive minutes before each

134 sampling or experiment. Following Barata et al. (2007), a dominance index (DI) was used to

135 quantify males' social rank. DI was calculated using the ratio between the number of dominant

136 behaviours (aggressive displays or nest-digging) and the number of dominant behaviours plus the

137 number of submissive behaviours (escape from an aggressive opponent).

138

139

Protocol for stimuli presentation

140

141

Twenty-two dominant males (standard lengths (SL) ranging from 6.5 to $10.9 \mathrm{~cm}$; mean $=$

$1429.3 \mathrm{~cm}$ ) were used to test behavioural responses to each sensory channel. They were placed in the 143 experimental aquarium 48 hours before the beginning of stimuli presentation. This study

144 consisted in six different treatments: Teleost Ringer solution (1), urine from dominant males (2),

145 real opponent in the jar (3), agonistic video playback (4), agonistic sound playback (5) and

146 holding water from dominant males (6). In experiment 1, fourteen fish received treatment 1 to 5

147 (one treatment per day) on consecutive days in a random order. In experiment 2, nine fish were

148 exposed to treatment 6 only. Experiment 2 was designed to develop another chemical stimulus

149 (holding water) to achieve the best bioassay (holding water or urine) to test this channel. Holding 150 water was tested in a separate experiment from urine to avoid any confounding effects from any

151 potential chemical cues that lingered in the test tank. Different males were used in the two

152 experiments except for one male that was used in both. After experiments, all subject males 
153 returned to their original tank and the whole set up was rinsed with water mixed with white

154 vinegar to eliminate any potential chemical cues in the aquarium, before new males were placed

155 in the experimental tanks.

156 Each experiment lasted 15 min. Five minutes before the beginning of the test, filter,

157 aeration, and internal heater were switched off. The visual contact with the social group was

158 interrupted through the Samsung's 172x LCD 17" monitor used during video playback. The

159 screen was switched on but covered by a removable opaque partition. At the same time, the

160 loudspeaker was switched on and the real plastic tube for chemical presentation replaced the lure

161 when necessary. The experiment started with a five minutes control period while no stimulus

162 was presented. This was followed by a ten minutes period of stimulus presentation, in which

163 either sound, chemical, or visual stimulation occurred. Details of the treatments were as follows:

164

165 Treatment 1. Teleost Ringer solution: $175 \mu \mathrm{L}$ of Ringer solution (Fresh water Teleost Ringer 166 composition was obtained from the Biological Bulletin Compendia website) was presented four

167 times during the stimulus presentation, when the subject fish approached or touched the pump's 168 tube.

169

170 Treatment 2. Urine from dominant males: After assessing the fish's social rank, urine was

171 collected by gently squeezing the abdomen from the anterior area to the genital papilla,

172 following the method described in Barata et al. (2007). Once sampled, the fish was immediately

173 placed back in its community tank. The urine from each male was stored at $-80{ }^{\circ} \mathrm{C}$ until use for

174 the experiments. The collected amounts of urine from dominant males (DI $>0.7)$ ranged from 5

$175 \mu \mathrm{L}$ to $340 \mu \mathrm{L}$ with a mean of $97 \mu \mathrm{L}$. The chemical composition of the urine was not analysed. 

solution) was delivered four times when the fish approached or touched the pump's tube.

178 Samples came from the same dominant male, but collected at least on two different dates, and 179 were randomly assigned from six different dominant males (DI $>0.7$ ). To avoid any familiar 180 effect, the urine came from a male unknown to the subject male, i.e. from a different community 181 tank.

182

183 Treatment 3. Real opponent in the jar: a male, intermediate or dominant (DI $>0.3)$, matched in 184 size with the subject, was introduced in the jar located inside the subject's tank, for ten minutes 185 of presentation without previous acclimatization.

187 Treatment 4. Agonistic video playback: Video playback consisted of three sequences from the 188 same stimulus male: two different biting sequences and one swimming sequence. The two biting 189 sequences were each composed of two bites and lasted five seconds. All sequences (biting and swimming) were recorded with a Logitech c 910 webcam (Logitech HD Pro c910, 1080p) and played back interactively (see below). They were filmed frontally through a one-way mirror associated to a LED light (LED light, wave 5W, TETRA, France) for the biting sequences and a $11 \mathrm{~W} 230 \mathrm{~V}$ light bulb for the swimming sequence. Both biting and swimming sequences were 194 randomly selected from four males with SL ranging from 7.1 to $10.7 \mathrm{~cm}(\mathrm{mean}=9.4 \mathrm{~cm})$. To avoid any familiar effect, fish used to create the video playback came from different tanks than the subjects. The stimulus male and subject male were matched in size (i.e. SL ratio $\leq 7 \%$ ). swimming sequence was immediately played back. As soon as the subject turned to the screen, 
199 one of the two biting sequences was played back. If the individual stayed in that position, the

200 second biting sequence was played back and so on until the two different biting sequences were

201 played back five times each. As soon as the tested fish stopped looking at the screen, the

202 swimming sequence was put on the screen.

203

204 Treatment 5. Agonistic sound playback: Agonistic sounds were played back (115 dB re. $1 \mu \mathrm{Pa}$ 205 at $3.8 \mathrm{~cm}$, within the range of the natural male sounds) with custom-made underwater 206 loudspeakers (frequency response $\pm 1.5 \mathrm{~dB}$ in the range $20 \mathrm{~Hz}-2 \mathrm{kHz}$ ) and a driver developed 207 by Fonseca \& Alves (2012). Sound stimuli were fed to the driver by a D/A converter (Edirol 208 UA-25, Roland, Japan) controlled by Adobe Audition 3.0 (Adobe Systems Inc., Mountain View, 209 CA, USA) on a laptop.

210 Two agonistic sounds of the same male were used as stimuli per experiment. These 211 sounds were randomly assigned from six different males with SL ranging $6.0 \mathrm{~cm}$ to $10.0 \mathrm{~cm}$ 212 (mean $=9.4 \mathrm{~cm})$ from the ENES sound archive (see Bertucci et al. (2012)) and from new 213 recordings made at the beginning of the study. All agonistic sounds presented 7-9 pulses (mean =

2148.0 pulses) and a pulse rate of $18 \pm 7$ pulses per second. The pulses mean dominant frequency of 215 the sounds was $289 \mathrm{~Hz} \pm 133 \mathrm{~Hz}$ (see Bertucci et al. (2012), for the description of sounds 216 analysis). Pulses dominant frequencies were obtained with the 'dfreq' function from the 217 'seewave' R package (Sueur, Aubin \& Simonis, 2008). As in treatment 4, playback was made 218 interactively, simulating close interactions with a potential opponent. The stimulus was played 219 back only when the tested individual approached the loudspeaker area, i.e. within 1 to $5 \mathrm{~cm}$ in 220 front of the loudspeaker. Each sound was played back five times in a random order, which 221 represented ten agonistic sounds in a test. 
223 Treatment 6. Holding water from dominant males: After quantifying the social rank, dominant 224 males $(\mathrm{Di}>0.7)$ were selected and placed individually in a jar containing two litres of aerated 225 water at $25 \pm 1{ }^{\circ} \mathrm{C}$ during three hours. One litre of this solution was used during chemical 226 presentation in the same day, to avoid any chemical degradation. We can assume the presence of 227 urine in the holding water. Indeed, for the purposes of another ongoing study on chemical 228 communication in the same fish, we injected three males with patent blue V (Prolabo, France). 229 Those males with an unknown status were small, median and large fish. Thirty minutes after the 230 injection, we observed that fish were urinating blue and three hours after, we noted a complete 231 blue coloration of the 2L-aquarium's water. This demonstrates that the fish urinated during those 232 three hours.

For this treatment, one litre of holding water was delivered continuously during the ten minutes of stimulus presentation. Holding water was obtained from eight different dominant males $(\mathrm{Di}>0.7)$ and the water from one stimulus male was randomly assigned to subject males. As for urine, the holding water used came from a male unknown to the subject male.

\section{Behavioural analysis}

Videos were analysed using JWatcher software (v. 1.0). The aggressive behaviours (i.e.

241 lateral displays, quivers, bites, darts and sounds produced) were counted and then summed to 242 produce an aggressive score (Simões et al., 2008). We quantified the number of up-and-down 243 swimming (when a territorial fish was swimming up and down against the wall of the aquarium).

244 We also quantified the tank's exploration score, for which the aquarium was artificially divided 
245 in three equal zones and the number of shifts between the three zones was counted. Finally, the

246 nest maintenance behaviour (when a territorial fish was moving sand within its territory) and the

247 number of the shelter's visit were also counted.

248

249

250

251

252

253

254

255

256

257 258

259

260

261

262

263

264

265

266

267

\section{Statistics}

All statistical tests were performed using R software (R Core Team, 2016). For each experiment, we analysed the influence of the treatments on five selected behaviours (agonistic, up-and-down swimming, tank's exploration, maintenance and the shelter's visit) separately.

Generalized linear mixed models ('glmer' functions from the 'Ime4' R package, Bates et al. (2014)) were used to assess the effect of the different treatments on the five selected behaviours. For models with Poisson family, overdispersion was tested, and if the model presented overdispersion we used a negative binomial model ('glmmADMB' R package, Skaug et al. (2013)) .

The following model was applied in experiment 1 for the five selected behaviours: Model $1<$ - Behaviour score $\sim$ treatment $*$ period + offset $(\log ($ Recording duration $))+(1 \mid$ day $)+(1 \mid$ subject)

And the following model was applied in experiment 2 for the five selected behaviours: Model 2 $<-$ Behaviour score $\sim$ period + offset $(\log ($ Recording duration $))+(1 \mid$ subject $)$

Model 1 tested the two interacting factors (treatment and period). The variable treatment had five levels. For both models, the variable period had two levels: the control and the stimulus presentation. Two random factors were added to the models: the day of the experimentation (1| day) for Model 1 and the subject identity (1| subject) for Model 1 and 2. An 'offset' took into 
268 account the different recording durations between the periods of control and stimulus 269 presentation. P-values were assessed using the 'Anova' function ('car' R package, Fox \& 270 Weisberg (2011)). In Model 1, if the effect of the variable treatment alone or the effect of the 271 interaction between factors was significant, post hoc tests using Tukey's adjustment were 272 performed using 'lsmeans' function ('Ismeans' R package, Lenth (2016)).

273

274 Ethical Note

275

276

All procedures described in this manuscript were conducted in accordance with 277 appropriate French national guidelines, permits and regulations regarding animal care and 278 experimental use (Approval $\mathrm{n}^{\circ} \mathrm{C} 42-218-0901$, ENES lab agreement, Direction Départementale 279 de la Protection des Populations, Préfecture du Rhône).

282 Results

\section{Experiment 1 - effect of chemical, visual and acoustic stimuli}

The interaction between the treatment and the trial period had an impact on agonistic

behaviour (Fig. 2 A). The view of a real opponent increased the number of agonistic behaviours observed during the stimulus presentation compared to the control period where no visual stimulus was present (Real opponent: $\mathrm{z}=4.252, P<0.001$ ). Moreover, the number of agonistic behaviours during the presentation of a real opponent was significantly higher than the number 
291 of agonistic behaviours observed during the presentation of all the other treatments (Teleost 292 Ringer solution: $\mathrm{z}=3.378, P=0.005$; Urine from dominant males: $\mathrm{z}=3.300, P=0.005$; 293 Agonistic video: $\mathrm{z}=4.628, P<0.001$; and Agonistic sound: $\mathrm{z}=3.334, P=0.005)$.

294 The interaction between the treatment and the trial period also had an impact on the up 295 and down swimming (Fig. 2 B). The view of a real opponent significantly decreased the number 296 of up and down swimming during the stimulus presentation in comparison with the presentation 297 of three other treatments: teleost Ringer solution $(\mathrm{z}=-2.750, P=0.051)$, dominant males' urine $298(\mathrm{z}=-3.074, P=0.051)$ and agonistic sound $(\mathrm{z}=-2.745, P=0.051)$.

299 When a real opponent was present, individuals had the tendency to increase tank's 300 exploration during the stimulus presentation in comparison with the control period where no 301 opponent could be seen (Fig. $2 \mathrm{C}, \mathrm{z}=3.058, P=0.056$ ).

Finally, we did not find any evidence that the number of shelter's visits (Fig. 2 D, $\chi 2=$ 303 2.055, $P=0.726$ ) and maintenance behaviour (Fig. $2 \mathbf{E}, \chi 2=8.292, P=0.081$ ) were influenced 304 by treatment or trial period.

305

306

\section{Experiment 2 - effect of holding water}

307

308

Holding water had no significant effect on agonistic behaviour (Fig. $3 \mathbf{A}, \chi 2=0.542, P=$ $=9.161, P=0.002)$, tended to increase the tank's exploration (Fig. 3

C, $\chi 2=2.922, P=0.087)$

311 but decreased significantly the number of visits to the shelter (Fig. $3 \mathbf{D}, \chi 2=8.605, P=0.003$ ).

312 We found no evidence that maintenance behaviour (Fig. $3 \mathbf{E}, \chi 2=0.657, P=0.418$ ) was 313 influenced by holding water. 
316

317

318

319

320

321

322

323

324

325

326

327

328

329

330

331

332

333

334

335 336

\section{Discussion}

Our study aimed to test behavioural responses during agonistic interactions to unimodal chemical, visual or acoustic signals in a social fish and to optimise a bioassay to study multimodal communication. Here we showed that only the visual channel elicited changes in the behaviour of dominant M. zebra males, including aggression and general activity (explorative behaviour and swimming up and down).

\section{Unimodal signals}

We found that the view of a real opponent provoked a highly aggressive reaction from the subject male. It also tended to increase the tank's exploration, which could be related to the subject attempting to reach the opponent confined in a jar. Subject males interacted with the intruder from three sides of the jar, ending up making more shifts between the aquarium zones. In addition, probably because they interacted for long periods with the intruder, the number of up and down swimming decreased. This aggressive response is in accordance with previous studies where visual cues alone were enough to elicit aggressive behaviour from the subject male. For example, in Bertucci et al. (2010), while an acoustic playback alone did not change the subject's behaviour in comparison to the control period in male $M$. zebra, the only view of a live contestant provoked a high aggressive answer from the tested male.

In the present study, all behavioural responses to unimodal chemical and acoustic treatments did not differ significantly from the control. Indeed, we did not find evidence that the tested fish reacted either to urine from dominant males, or to the playback of agonistic sounds 
337 presented alone. Concerning the acoustic treatment, this result is in accordance with previous

338 studies which also found no reaction to sound playback alone as in fish agonistic sounds are

339 typically produced within short distance from the receiver and associated with conspicuous

340 visual displays (Ladich \& Myrberg, 2006). Our first assumption was that acoustic stimulus

341 would induce indirect territorial behaviours such as increased activity and nest building. Myrberg

342 \& Riggio (1985) showed that sounds coming from nearest neighbour provoked less diving

343 displays of courtship than sounds coming from other males within the colony. But in M. zebra,

344 sounds are associated with behaviours such as quivers during close agonistic interactions

345 (Simões et al., 2008). It seems that sounds alone made by a contestant do not suffice to incite

346 territorial males to be involved in aggression or territorial activities. Butler \& Maruska (2015)

347 showed in the cichlid Astatotilapia burtoni that the lateral line plays an essential role for mutual

348 assessment of opponents during agonistic encounters. This system facilitates the use of non-

349 contact assessment. Additional vibrational information could be essential and the role of the

350 lateral line system during agonistic interactions in M. zebra clearly needs further investigation.

351 Regarding the chemical component, Chien (1973) has reported the effect of chemical cues in the

352 South American angelfish (Pterophyllum scalare) in a reproductive context. Chemical cues

353 emitted by males increased spawning rates in the females. By contrast, in our study, urine from

354 dominant males did not elicit any behavioural response from the tested males. To our best

355 knowledge this is the first time that isolated chemical stimuli are tested in an agonistic context in

356 fish. Other studies in fish underline that, during agonistic encounters, urine (like sound) is a

357 signal used in close range aggressive interactions and needs concurrent visual stimuli to elicit

358 immediate behavioural responses (Barata et al., 2007; Keller-Costa et al., 2016). 

unimodal signals in M. zebra into the proposed categories made by Partan \& Marler (1999).

361 Because chemical and acoustic stimuli did not elicit any response in $M$. zebra males but visual

362 stimuli did, unimodal signals should be categorized as non-redundant, since redundant signals

363 have equivalent effects on a receiver. When presented as a composite signal, non-redundant

364 signals can lead to different effects such as an independence effect. When combined, non365 redundant signals provoke behavioural reactions which are the same as those observed when

366 signals are presented alone. On the other hand, if one signal overshadows or changes the effect of

367 the other, it has a dominance or a modulation effect. If otherwise the combination of those

368 signals elicits an entirely new response there is an emergence effect. How different sensory 369 modalities interplay during fish communication, in particular during agonistic interactions, 370 remains to be unravelled. In $M$. zebra acoustic agonistic signals seem to modulate the response to 371 the visual channel (Bertucci et al., 2010). In contrast, the acoustic sensory modality seems to 372 dominate over the visual one in a small marine goby (Amorim et al., 2013). In Neolamprologus 373 pulcher chemical signals appear to modulate the response to visual signals during an agonistic 374 encounter (Bayani, Taborsky \& Frommen, 2017). Future work needs to address how the different 375 sensory channels interact to produce an adaptive response.

377 Which bioassay?

378 In addition to investigate the behavioural response to unimodal stimuli, we aimed to 379 develop a bioassay to assess the role of each sensory channel in eliciting behavioural decisions. 380 Different types of stimuli can be chosen to test the role of one signal modality with each having 381 different pros and cons. For example, in the present study a live opponent was the treatment that 
382 elicited the strongest response from subject males compared to a video playback. However,

383 because within one-modality a signal is usually multicomponent (Candolin, 2003), it is important

384 to be able to control the other parameters, apart from the ones that are being tested. Although we

385 controlled for size effects (live intruders were size-matched to the tested fish) we could not

386 control other aspects such as changes in body colour, behaviour, etc. Because of these

387 drawbacks, other studies have used different visual stimuli such as mirror tests (e.g. Raffinger \&

388 Ladich (2009)) or visual computer-manipulated stimuli (Watanabe, Shinozuka \& Kikusui, 2016).

389 Although more and more research uses computer animation as a tool to control visual cues,

390 including in cichlids (Balzarini et al., 2016), our results underlined an absence of response

391 towards video playback. This was recently the case in another study using cichlids

392 (Wackermannova et al., 2017). The use of RGB video screen, the screen frame rate (developed

393 for human sight), the lack of UV components and three-dimensional depth are constraints that

394 may affect visual perception and could explain this absence of response. In any case, natural 395 motion patterns, velocity and interaction should not have been affected here as video playbacks 396 consisted of recordings from real individuals either biting the tank's glass with a one-way mirror, 397 or performing routine swimming.

398 Finally, we tested another chemical cue, holding water from dominant males, which is 399 classically used in cichlids (de Caprona, 1974; Thünken et al., 2009). Here, holding water 400 increased the up and down swimming, tended to increase the tank's exploration and decreased 401 the number of shelter's visits. Unlike urine, holding water seems to make the fish aware of the 402 potential presence of a contestant even though no visual signal was present. Holding water 403 represents a more diffuse cue than urine, as urine is generally used during close range aggressive 404 interactions (Barata et al., 2007; Escobar-Camacho \& Carleton, 2015). It also contains in 
405 addition to urine, other components coming from the gills, the mucus, and the faeces. These 406 differences could explain that urine without visual signal had no effect on M. zebra males' 407 behaviours while holding water without visual signal had an impact. Consequently, the best 408 bioassay in testing behavioural answers in M. zebra to chemical signals seems to be the use of 409 holding water. It is a lot easier and less intrusive to collect than pure urine, where collected 410 amounts varied from nothing to very small quantities $(<340 \mu \mathrm{L})$. Moreover, the handling 411 provokes a stressful response from the handled fish, typically accompanied by increased 412 circulating cortisol (Ramsay et al., 2009). Because we left the fish in a two litres tank during 413 three hours to obtain holding water, the manipulation is less stressful and we can manage larger 414 liquid quantities. Nevertheless, if the question is to seek the existence of active molecules in 415 urine during agonistic encounters, then sampling pure urine should be more promising. In conclusion, M. zebra territorial males seem to rely mostly on the visual sensory

417 modality, as the view of a real opponent provoked intense aggressive reaction but the acoustic 418 signal and chemical cue alone did not elicit obvious changes in their immediate behaviours. The 419 different sensory channels seem to be non-redundant (sensu Partan \& Marler (1999)) in the study 420 species and likely in most other fishes (Ladich, 2004), but how different modalities are integrated 421 to produce adaptive behaviour in fish remains to be answered. Our results also emphasize the 422 need to optimise experimental protocols to test the significance of multimodal communication 423 during specific behavioural contexts, to better understand the evolution of signalling across 424 vertebrates (Partan \& Marler, 1999; Candolin, 2003; Narins et al., 2005).

427 We thank Friedrich Ladich and an anonymous referee for their valuable comments. 


\section{References}

430 Amorim MCP., da Ponte AN., Caiano M., Pedroso SS., Pereira R., Fonseca PJ. 2013. Mate

431 preference in the painted goby: the influence of visual and acoustic courtship signals. Journal of 432 Experimental Biology 216:3996-4004. DOI: 10.1242/jeb.088682.

433 Amorim MCP., Simões JM., Fonseca PJ., Turner GF. 2008. Species differences in courtship 434 acoustic signals among five Lake Malawi cichlid species (Pseudotropheus spp.). Journal of Fish 435 Biology 72:1355-1368. DOI: 10.1111/j.1095-8649.2008.01802.x.

436 Balzarini V., Taborsky M., Villa F., Frommen JG. 2016. Computer animations of color markings 437 reveal the function of visual threat signals in Neolamprologus pulcher. Current Zoology:zow086. 438 DOI: $10.1093 / \mathrm{cz} /$ zow086.

439 Barata EN., Hubbard PC., Almeida OG., Miranda A., Canário AV. 2007. Male urine signals 440 social rank in the Mozambique tilapia (Oreochromis mossambicus). BMC Biology 5:54. DOI: $441 \quad 10.1186 / 1741-7007-5-54$.

442 Bates D., Mächler M., Bolker B., Walker S. 2014. Fitting Linear Mixed-Effects Models using $443 \quad$ lme4.

444 Bayani D-M., Taborsky M., Frommen JG. 2017. To pee or not to pee: urine signals mediate 445 aggressive interactions in the cooperatively breeding cichlid Neolamprologus pulcher.

446 Behavioral Ecology and Sociobiology 71:37. DOI: 10.1007/s00265-016-2260-6.

447 Bertucci F., Attia J., Beauchaud M., Mathevon N. 2012. Sounds produced by the cichlid fish 448 Metriaclima zebra allow reliable estimation of size and provide information on individual 449 identity. Journal of Fish Biology 80:752-766. DOI: 10.1111/j.1095-8649.2012.03222.x. 450 Bertucci F., Beauchaud M., Attia J., Mathevon N. 2010. Sounds Modulate Males’ 
451 Aggressiveness in a Cichlid Fish: Sounds Modulate Males’ Aggressiveness in a Cichlid Fish. 452 Ethology 116:1179-1188. DOI: 10.1111/j.1439-0310.2010.01841.x.

453 Butler JM., Maruska KP. 2015. The mechanosensory lateral line is used to assess opponents and 454 mediate aggressive behaviors during territorial interactions in an African cichlid fish. Journal of 455 Experimental Biology 218:3284-3294. DOI: 10.1242/jeb.125948.

456 Candolin U. 2003. The use of multiple cues in mate choice. Biological Reviews 78:575-595. 457 DOI: $10.1017 / \mathrm{S} 1464793103006158$.

458 de Caprona M-DC. 1974. The effect of chemical stimuli from conspecifics on the behavior of 459 Haplochromis burtoni (Cichlidae, Pisces). Experientia 30:1394-1395. DOI:

$46010.1007 / \mathrm{BF} 01919654$.

461 Chen C-C., Fernald RD. 2011. Visual Information Alone Changes Behavior and Physiology 462 during Social Interactions in a Cichlid Fish (Astatotilapia burtoni). PLOS ONE 6:e20313. DOI: 463 10.1371/journal.pone.0020313.

464 Chien AK. 1973. Reproductive behaviour of the angelfish Pterophyllum scalare (Pisces:

465 Cichilidae) II. Influence of male stimuli upon the spawning rate of females. Animal Behaviour 466 21:457-463. DOI: 10.1016/S0003-3472(73)80005-3.

467 Darwin C., Prodger P. 1998. The Expression of the Emotions in Man and Animals. Oxford 468 University Press.

469 Dijkstra PD., Lindström J., Metcalfe NB., Hemelrijk CK., Brendel M., Seehausen O., Groothuis 470 TGG. 2010. Frequency-Dependent Social Dominance in a Color Polymorphic Cichlid Fish.

471 Evolution 64:2797-2807. DOI: 10.1111/j.1558-5646.2010.01046.x.

472 Escobar-Camacho D., Carleton KL. 2015. Sensory modalities in cichlid fish behavior. Current 473 Opinion in Behavioral Sciences 6:115-124. DOI: 10.1016/j.cobeha.2015.11.002. 
474 Estramil N., Bouton N., Verzijden MN., Hofker K., Riebel K., Slabbekoorn H. 2014. Cichlids 475 respond to conspecific sounds but females exhibit no phonotaxis without the presence of live 476 males. Ecology of Freshwater Fish 23:305-312. DOI: 10.1111/eff.12081.

477 Fonseca PJ., Alves JM. 2012. A new concept in underwater high fidelity low frequency sound 478 generation. Review of Scientific Instruments 83:055007. DOI: 10.1063/1.4717680.

479 Fox J., Weisberg S. 2011. Multivariate linear models in R. An R companion to applied 480 regression, 2nd edn. SAGE Publications.

481 Higham JP., Hebets EA. 2013. An introduction to multimodal communication. Behavioral 482 Ecology and Sociobiology 67:1381-1388. DOI: 10.1007/s00265-013-1590-x.

483 Holzberg S. 1978. A field and laboratory study of the behaviour and ecology of Pseudotropheus 484 zebra (Boulenger), an endemic cichlid of Lake Malawi (Pisces; Cichlidae) M1. Journal of 485 Zoological Systematics and Evolutionary Research 16:171-187. DOI: 10.1111/j.1439486 0469.1978.tb00929.x.

487 Hurd PL., Enquist M. 2001. Threat display in birds. Canadian Journal of Zoology 79:931-942. 488 DOI: $10.1139 / \mathrm{z} 01-062$.

489 Keller-Costa T., Canário AVM., Hubbard PC. 2015. Chemical communication in cichlids: A 490 mini-review. General and Comparative Endocrinology 221:64-74. DOI:

491 10.1016/j.ygcen.2015.01.001.

492 Keller-Costa T., Saraiva JL., Hubbard PC., Barata EN., Canário AVM. 2016. A Multi493 Component Pheromone in the Urine of Dominant Male Tilapia (Oreochromis mossambicus) 494 Reduces Aggression in Rivals. Journal of Chemical Ecology 42:173-182. DOI: 10.1007/s10886495 016-0668-0.

496 Knight ME., Turner GF. 1999. Reproductive isolation among closely related Lake Malawi 
497 cichlids: can males recognize conspecific females by visual cues? Animal Behaviour 58:761498 768. DOI: 10.1006/anbe.1999.1206.

499 Kobayashi M. 2002. Hormonal and pheromonal control of spawning behavior in the goldfish.

500 Fish Physiology and Biochemistry 26:71-84. DOI: 10.1023/A:1023375931734.

501 Ladich F. 2004. Sound Production and Acoustic Communication. In: von der Emde, Mogdans J, 502 Kapoor BG eds. The Senses of Fish. Kluwer Academic Publishers \& Narosa Publishing House, 503 New Delhi, 210-230. DOI: 10.1007/978-94-007-1060-3_10.

504 Ladich F., Myrberg AA. 2006. Agonistic behavior and acoustic communication. In 505 Communication in fishes. Vol 1 (ed. F. Ladich, S.P. Collin, P. Moller and B. G. Kapoor), 121506 148, Enfield, NH: Science Publishers.

507 Lenth RV. 2016. Least-squares means: the R package lsmeans. J Stat Softw 69:1-33.

508 Longrie N., Poncin P., Denoël M., Gennotte V., Delcourt J., Parmentier E. 2013. Behaviours 509 Associated with Acoustic Communication in Nile Tilapia (Oreochromis niloticus). PLoS ONE 510 8:e61467. DOI: 10.1371/journal.pone.0061467.

511 Maruska KP., Fernald RD. 2012. Contextual chemosensory urine signaling in an African cichlid 512 fish. Journal of Experimental Biology 215:68-74. DOI: 10.1242/jeb.062794.

513 Maruska KP., Ung US., Fernald RD. 2012. The African Cichlid Fish Astatotilapia burtoni Uses

514 Acoustic Communication for Reproduction: Sound Production, Hearing, and Behavioral

515 Significance. PLOS ONE 7:e37612. DOI: 10.1371/journal.pone.0037612.

516 Mellor D., Wilt L., Gershenson D., Howe D., Jordan R. 2012. Female preference in the context 517 of male-male interactions in Maylandia zebra of Lake Malawi. Journal of Ethology 30:181-186. 518 DOI: $10.1007 / \mathrm{s} 10164-011-0289-6$.

519 Myrberg jr AA., Riggio RJ. 1985. Acoustically mediated individual recognition by a coral reef 
520 fish (Pomacentrus partitus). Animal Behaviour 33:411-416. DOI: 10.1016/S0003-

$521 \quad 3472(85) 80065-8$.

522 Narins PM., Grabul DS., Soma KK., Gaucher P., Hödl W. 2005. Cross-modal integration in a

523 dart-poison frog. Proceedings of the National Academy of Sciences of the United States of

524 America 102:2425-2429. DOI: 10.1073/pnas.0406407102.

525 Partan S., Marler P. 1999. Communication Goes Multimodal. Science 283:1272-1273. DOI:

$52610.1126 /$ science.283.5406.1272.

527 Partan SR., Marler P. 2005. Issues in the Classification of Multimodal Communication Signals.

528 The American Naturalist 166:231-245. DOI: 10.1086/431246.

529 Plenderleith M., Oosterhout C van., Robinson RL., Turner GF. 2005. Female preference for 530 conspecific males based on olfactory cues in a Lake Malawi cichlid fish. Biology Letters 1:411531 414. DOI: $10.1098 / \mathrm{rsbl} .2005 .0355$.

532 R Core Team. 2016. R: A Language and Environment for Statistical Computing. Vienna, 533 Austria: R Foundation for Statistical Computing.

534 Raffinger E., Ladich F. 2009. Acoustic threat displays and agonistic behaviour in the red-finned 535 loach Yasuhikotakia modesta. Journal of Ethology 27:239-247. DOI: 10.1007/s10164-008-01095369.

537 Ramsay JM., Feist GW., Varga ZM., Westerfield M., Kent ML., Schreck CB. 2009. Whole-body 538 cortisol response of zebrafish to acute net handling stress. Aquaculture (Amsterdam, 539 Netherlands) 297:157-162.

540 Simões J., Duarte IG., Fonseca PJ., Turner GF., Clara Amorim M. 2008. Courtship and agonistic

541 sounds by the cichlid fish Pseudotropheus zebra. The Journal of the Acoustical Society of

542 America 124:1332. DOI: 10.1121/1.2945712. 
543 Skaug H., Fournier D., Nielsen A., Magnusson A., Bolker B. 2013. Generalized linear mixed

544 models using AD model builder. R package version 0.77.

545 Smith CL., Evans CS. 2013. A new heuristic for capturing the complexity of multimodal signals.

546 Behavioral Ecology and Sociobiology 67:1389-1398. DOI: 10.1007/s00265-013-1490-0.

547 van Staaden MJ., Searcy WA., Hanlon RT. 2011. 3 Signaling Aggression. Advances in genetics

$548 \quad 75: 23$

549 Sueur J., Aubin T., Simonis C. 2008. Seewave, a Free Modular Tool for Sound Analysis and

550 Synthesis. Bioacoustics 18:213-226. DOI: 10.1080/09524622.2008.9753600.

551 Thünken T., Waltschyk N., Bakker TCM., Kullmann H. 2009. Olfactory self-recognition in a

552 cichlid fish. Animal Cognition 12:717-724. DOI: 10.1007/s10071-009-0231-2.

553 Wackermannova MA., Horky P., Amorim MCP., Fonseca PJ. 2017. Computer-manipulated

554 stimuli as a research tool in Mozambique tilapia Oreochromis mossambicus. acta ethologica:1-

555 10. DOI: $10.1007 / \mathrm{s} 10211-017-0252-9$.

556 Watanabe S., Shinozuka K., Kikusui T. 2016. Preference for and discrimination of videos of

557 conspecific social behavior in mice. Animal Cognition 19:523-531. DOI: 10.1007/s10071-016-

558 0953-X.

559 


\section{Figure 1 (on next page)}

General experimental design.

Dominant males were placed in the experimental aquarium forty-eight hours before the beginning of the experiment. First, the visual contact with the social group was interrupted through the LCD screen. The screen was switched on and was behind a removable opaque partition (no image was presented). Trials started with the control period (without stimulus) during five minutes, followed by the stimuli presentation during ten minutes: (1) Teleost Ringer solution, (2) urine from dominant males, (3) real opponent in the jar, (4) agonistic video playback, (5) agonistic sound playback or (6) holding water from dominant males. In experiment 1, fish received treatment (1) to (5) (one treatment per day) on consecutive days in a random order. In experiment 2, fish were exposed to treatment (6) only. 


\section{PeerJ}

Manuscript to be reviewed

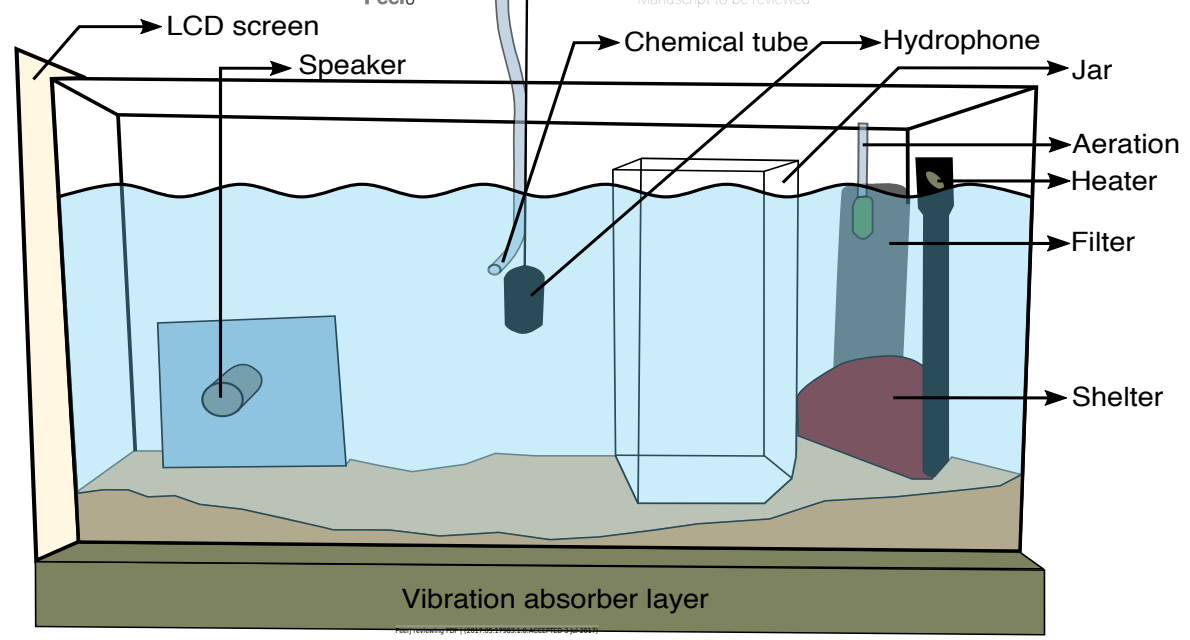




\section{Figure 2 (on next page)}

Effect of the treatments on five selected behaviours.

Effect of the treatments (Teleost Ringer solution, urine from dominant males, real opponent, agonistic video playback or agonistic sound playback) on the number of $\mathbf{A}$ ) agonistic behaviours, B) up and down swimming, C) tank's exploration, D) shelter's visit, and E) maintenance. Each treatment included two periods, the control period without stimulus (in black) and the stimuli presentation (in light grey). Beanplots ('beanplot' R package (Kampstra, 2008) ) combine individual observations (white lines), dataset distribution, the overall dataset average (dashed horizontal line) and the average for each subset (heavy horizontal black line). ${ }^{+P} \leq 0.1 ; * P \leq 0.05 ;{ }^{* * P} \leq 0.01,{ }^{* * * P} \leq 0.001$. 


\section{Figure 3 (on next page)}

Effect of the holding water on five selected behaviours.

Effect of the holding water from dominant males on the number of $\mathbf{A}$ ) agonistic behaviours, B) up and down swimming, C) tank's exploration, D) shelter's visit, and E) maintenance, during the control period without stimulus (in black) and the stimulus presentation (in light grey). Beanplots ('beanplot' R package (Kampstra, 2008)) combine individual observations (white lines), dataset distribution, the overall dataset average (dashed horizontal line) and the average for each subset (heavy horizontal black line). ${ }^{\mathrm{t}} \mathrm{P} \leq 0.1$; **P $\leq 0.01$. 

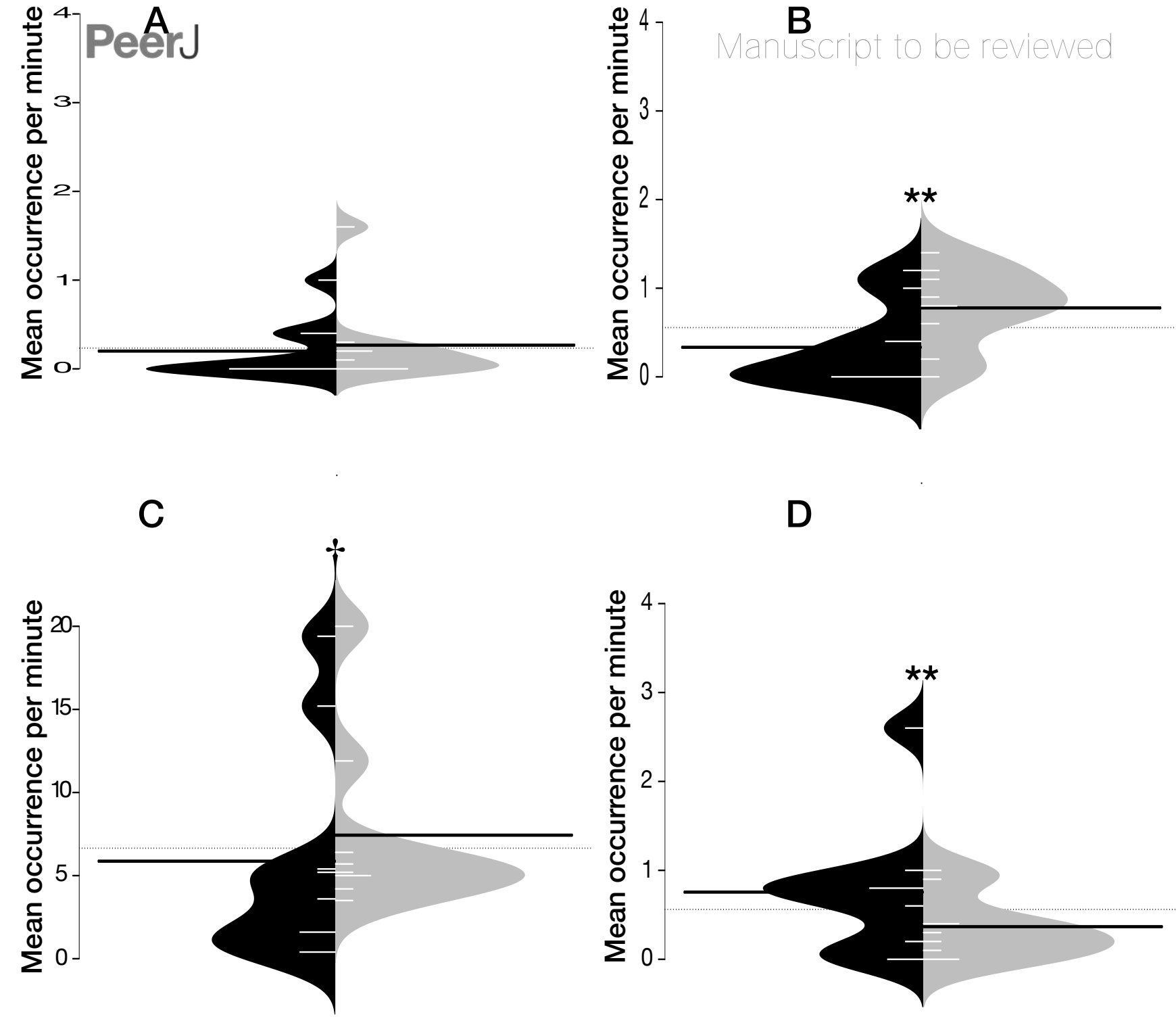
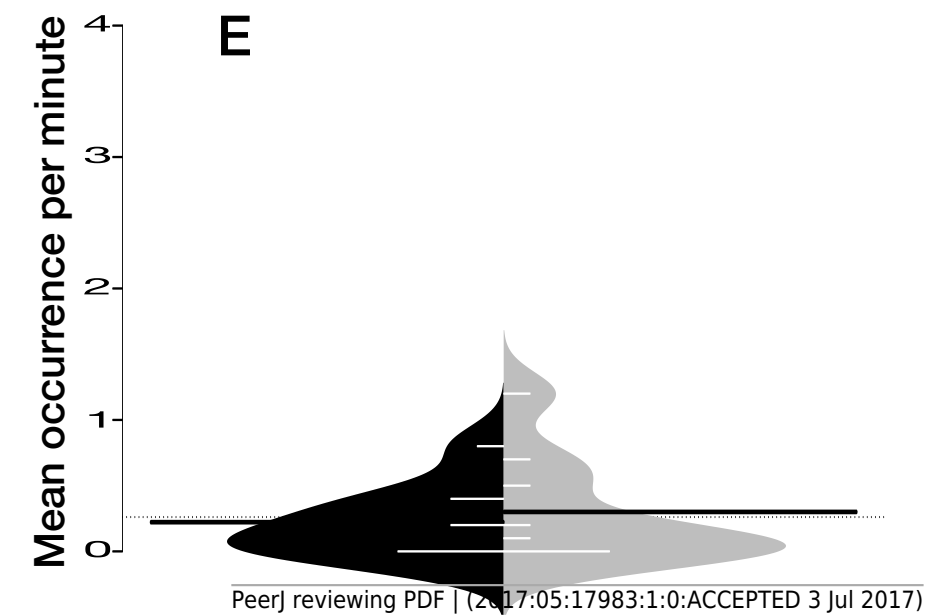

- Control (no stimulus)

Stimulus presentation (holding water from dominant males) 